\title{
ESTRATEGIAS DE ADAPTACIÓN AL CAMBIO CLIMÁTICO EN DOS COMUNIDADES RURALES DE MÉXICO Y EL SALVADOR
}

\author{
Minerva Campos ${ }^{1}$ \\ Doribel Herrador ${ }^{2}$ \\ Carlos Manuel ${ }^{2}$ \\ Michael K. McCall' ${ }^{13}$ \\ ${ }^{1}$ Centro de Investigaciones en Geografía Ambiental. Universidad Nacional Autónoma de México \\ ${ }^{2}$ Departamento de Humanidades: Historia, Geografía y Arte. Universidad Carlos III de Madrid \\ cmanuel@hum.uc3m.es \\ ${ }^{3}$ Faculty of Geo-Information Science and Earth Observation (ITC). University of Twente, Enschede, The Netherlands
}

\section{RESUMEN}

En este artículo se exploran las estrategias de adaptación local para hacer frente a las perturbaciones relacionadas con el cambio climático mediante dos estudios de caso en zonas rurales de México y El Salvador. Además, los resultados son discutidos en el contexto de la intersección entre las respuestas locales y el papel de las iniciativas institucionales y políticas públicas, las cuales son determinantes para estructurar la efectividad de las respuestas locales a largo plazo.

Palabras clave: adaptación, cambio climático, capacidades locales, México, El Salvador.

\section{ABSTRACT}

This article explores local adaptation strategies to cope with impacts related to climate change through the analysis of two case studies in rural Mexico and El Salvador. Furthermore, the results are discussed in the context of the intersection between local responses and the role of institutional and public policy initiatives, which are crucial for structuring the effectiveness of long-term local responses.

Key words: adaptation, climate change, local capacities, Mexico, El Salvador.

Fecha de recepción: junio 2011.

Fecha de aceptación: diciembre 2012. 


\section{INTRODUCCIÓN}

Existe la certeza de que el clima mundial está y seguirá cambiando a un ritmo y con una intensidad sin precedentes en la historia humana reciente (IPCC, 2007). Ante los impactos asociados al cambio y la variabilidad climática están surgiendo, desde la escala local, diversas estrategias como respuesta a tales cambios (Mertz et al., 2009). La diferencia fundamental entre cambio climático y variabilidad climática radica en que el primero incluye alteraciones a largo plazo y a escala global, cuyas causas pueden ser de origen natural o antropogénico; mientras que la variabilidad climática se refiere a las fluctuaciones a corto plazo y asociadas a condiciones meteorológicas naturales y propias de cada región (Hageback et al., 2005). Sin embargo, se reconoce la influencia que el cambio climático tiene sobre la variabilidad climática, por lo que no es posible establecer impactos diferenciados a escala local (Smith et al., 2000). En este artículo nos referiremos a cambio y variabilidad climática simultáneamente, sin entrar a determinar el origen de los mismos, sino centrando el interés en el análisis de las respuestas que, ante esos impactos, ofrece la población local de dos regiones pertenecientes a países en desarrollo.

Los países en desarrollo son más vulnerables a los cambios climáticos que los países desarrollados debido a una mayor exposición a eventos hidrometeorológicos extremos (EHE), unas infraestructuras deficientes y al menor capital para el desarrollo y la difusión de medidas de adaptación (Fischer et al., 2005; Tubiello y Rosenzweig, 2008). La mayoría de los impactos de cualquier alteración climática afectarán especialmente a los grupos que dependen más directamente de los recursos naturales, como es el caso de los pequeños agricultores en los países en desarrollo (Adger, 2000; Morton, 2007). Debido a que las condiciones climáticas extremas y los impactos asociados tienden a acentuar los desafíos sociales y económicos actuales (Cannon, 1994), y teniendo en cuenta la velocidad a la que los cambios están ocurriendo, es urgente conocer si los grupos más vulnerables responden y se adaptan de manera exitosa a los nuevos escenarios climáticos (Berkes y Jolly, 2001; Adger et al., 2005; Eakin et al., 2006; Bryan et al., 2009).

Para los propósitos de este trabajo entendemos la capacidad de adaptación como los ajustes del sistema social en respuesta a estímulos climáticos, o sus impactos, de modo que reduzcan el daño causado y que potencien las oportunidades locales (Adger, 2000; Landa et al., 2008). Las adaptaciones no están aisladas de otras decisiones, y se producen en el contexto del cambio demográfico, cultural y económico, así como en un escenario de transformaciones en las tecnologías de la información, la gobernanza y los flujos del capital (O’Brien y Leichenko, 2000). Walker y otros (2002) sostienen que la capacidad de adaptación refleja el aprendizaje, la capacidad de experimentar con flexibilidad y de adoptar nuevas soluciones, así como el desarrollo de respuestas generalizadas (a nivel individual o colectivo) para amplias clases de desafíos. La adaptación al cambio climático emerge como un concepto clave en la investigación sobre cambio climático, ya que a través de la capacidad de respuesta y los mecanismos de adaptación es posible conocer si los sistemas sociales están siendo resistentes a los impactos y a los cambios climáticos. Conocer las respuestas locales para afrontar los retos ambientales resulta esencial para la planificación a largo plazo, ya que en la formulación de políticas es más efectivo tener una clara comprensión de los escenarios de cambio climático local y regional, así como de 
las estrategias y capacidades locales de adaptación (Eakin et al., 2006; Mertz et al., 2009; Eriksen y Selboe, 2012).

En este artículo exploramos las respuestas locales para hacer frente a las perturbaciones relacionadas con el cambio climático mediante dos casos de estudio en zonas rurales de México y El Salvador. A pesar de las evidentes diferencias entre ambos países, los casos de estudio presentados en este trabajo comparten varios aspectos comunes: primero, que en ambos casos, la base de la economía y subsistencia rural es agrícola y se centra en el cultivo del maíz, por lo que son comunidades altamente dependientes de los factores climáticos y de su variabilidad; segundo, que en ambos casos los sistemas de organización social son determinantes en la gestión del territorio, en las formas de aprovechamiento y, por ende, en la generación de respuestas conjuntas para hacer frente a los desafíos ambientales; y tercero, que aunque a diferente escala, ambos se encuentran en contextos de importantes cambios estructurales debido a los efectos de la globalización y las políticas neoliberales, lo cual también ha mermado su capacidad para resolver y enfrentar los retos ambientales actuales. Ambos países se encuentran en una de las regiones más vulnerables de América Latina ante los efectos del cambio climático, por estar localizada dentro de una franja de huracanes y zonas costeras bajas, entre otras particularidades (IPCC, 2007; Conde-Álvarez y SaldañaZorrilla, 2007; Samaniego, 2009).

Este trabajo tiene como principal objetivo identificar las respuestas locales, estrategias y medidas de adaptación ante los retos ambientales que afectan a la población en los dos ámbitos de estudio; además, se discute la intersección entre estas respuestas locales y las diferentes iniciativas institucionales y políticas públicas que existen en cada caso y que surgen a escala supra-local. A fin de cuentas, se considera que un correcto análisis de dicha intersección puede facilitar la reorientación o la propuesta de nuevas actuaciones que contribuyan a fortalecer la capacidad de respuesta local ante las amenazas ambientales.

\section{CASOS DE ESTUDIO: LA RESPUESTA LOCAL ANTE EL CAMBIO CLIMÁTICO}

Los casos de estudio descritos a continuación presentan un análisis a escala local de las estrategias de adaptación al cambio climático que la misma población está desarrollando. Se analizan dos lugares específicos: en México, el ejido de Ichamio, en el municipio de La Huacana (estado de Michoacán); y en El Salvador, el Área Natural de Cinquera, que comprende parte de los Municipios de Cinquera, Tejutepeque, Suchitoto, Tenancingo y Jutiapa (departamentos de Cabañas y Cuscatlán). La tabla 1 muestra algunas características básicas de ambos casos de estudio, en donde se puede apreciar la existencia de rasgos comunes, como son los altos índices de pobreza, la agricultura como principal medio de vida, el cultivo del maíz como base de la alimentación, el manejo de áreas de bosque en forma colectiva y, además, unas condiciones climáticas similares. 
Tabla 1

CARACTERIZACIÓN DE LAS ZONAS DE ESTUDIO EN MÉXICO Y EL SALVADOR

\begin{tabular}{|c|c|c|}
\hline Características & México & El Salvador \\
\hline $\begin{array}{l}\text { Entidad Federativa/ Depar- } \\
\text { tamento }\end{array}$ & Michoacán & Cabañas \\
\hline Municipio & La Huacana & $\begin{array}{l}\text { Cinquera, Tejutepeque y Su- } \\
\text { chitoto }\end{array}$ \\
\hline Nombre de la comunidad & Ichamio & $\begin{array}{l}\text { Caseríos: Cinquera, San Nico- } \\
\text { lás, Pepeistenango, El Tule, El } \\
\text { Cacao, San Benito y El Calva- } \\
\text { rio (Municipio de Cinquera), } \\
\text { Copapyo (Municipio de Suchi- } \\
\text { toto), San Francisco Echeverría } \\
\text { (Municipio de Tejutepeque) }\end{array}$ \\
\hline Localización & $\begin{array}{l}18^{\circ} 57^{\prime} 07^{\prime \prime} \mathrm{N} \\
101^{\circ} 49^{\prime} 25^{\prime} \mathrm{O}\end{array}$ & $\begin{array}{l}13^{\circ} 53^{\prime} 1.78 » \mathrm{~N} \\
88^{\circ} 57^{\prime} 49.00 » \mathrm{O}\end{array}$ \\
\hline Superficie total (ha) & $5.564,87$ ha & 5.300 ha \\
\hline Clima & $\begin{array}{l}\text { Aw: Cálido subhúmedo con } \\
\text { lluvias en verano }\end{array}$ & $\begin{array}{l}\text { Aw: Cálido subhúmedo con } \\
\text { lluvias en verano }\end{array}$ \\
\hline Tipos de vegetación & $\begin{array}{l}\text { Bosque tropical caducifolio } \\
\text { Bosque tropical subcaducifolio } \\
\text { Bosque de coníferas (Quercus } \\
\text { sp.) }\end{array}$ & Bosque seco tropical \\
\hline Áreas Naturales Protegidas & $\begin{array}{l}\text { Parte del ejido forma parte } \\
\text { de la Reserva de la Biosfera } \\
\text { Zicuirán-Infiernillo (Decreto } \\
\text { 2007) }\end{array}$ & $\begin{array}{l}\text { Área Natural de Cinquera (for- } \\
\text { ma parte del Sistema Nacional } \\
\text { de Áreas Protegidas) }\end{array}$ \\
\hline $\begin{array}{l}\text { Número total de comuneros/ } \\
\text { ejidatarios/ propietarios }\end{array}$ & 98 & 200 \\
\hline Cultivos principales & $\begin{array}{l}\text { Maíz, sorgo, cacahuate, ajonjo- } \\
\text { lí, jamaica }\end{array}$ & $\begin{array}{l}\text { Maíz, frijol, hortalizas y fruta- } \\
\text { les (mango, aguacate, cítricos, } \\
\text { guayaba) }\end{array}$ \\
\hline $\begin{array}{l}\text { Número de muestra (partici- } \\
\text { pantes talleres) }\end{array}$ & 31 & 42 \\
\hline $\begin{array}{l}\text { Tipo de propiedad de la tie- } \\
\text { rra (participantes talleres) }\end{array}$ & $\begin{array}{l}\text { Ejidal: } \\
30 \text { propietarios } \\
1 \text { arrendatario } \\
\end{array}$ & $\begin{array}{l}\text { Privada: } \\
26 \text { propietarios } \\
16 \text { arrendatarios } \\
\end{array}$ \\
\hline Sexo (participantes talleres) & $\begin{array}{l}25 \text { hombres } \\
6 \text { mujeres }\end{array}$ & $\begin{array}{l}28 \text { hombres } \\
14 \text { mujeres }\end{array}$ \\
\hline $\begin{array}{l}\text { Tamaño de parcela agrícola } \\
\text { (participantes talleres) }\end{array}$ & $\begin{array}{l}<10 \text { ha }(10) \\
11-30 \text { ha }(11) \\
>30 \text { ha }(10)\end{array}$ & $\begin{array}{l}0,5-0,7 \text { ha }(21) \\
1,5-3,0 \text { ha }(16) \\
3,5-4,0 \text { ha }(5)\end{array}$ \\
\hline
\end{tabular}

Fuente: Elaboración propia. 


\subsection{Variabilidad climática en Ichamio (México) y Cinquera (El Salvador)}

A partir del análisis de los patrones climáticos en diferentes estaciones meteorológicas a lo largo de América Central y el norte de Suramérica, Aguilar y otros (2005) revelan una variedad de cambios en los valores extremos de temperatura y precipitación durante las últimas décadas (1961-2003), con incrementos en las temperaturas extremas que oscilan, aproximadamente, entre los $0,2^{\circ} \mathrm{C}$ y $0,3^{\circ} \mathrm{C}$ por década.

Para el caso de México, los datos climáticos del área de estudio sobre precipitación y temperatura para los últimos 43 años corresponden a la estación Infiernillo, de la Comisión Federal de Electricidad. El análisis de estos datos muestra que ambas variables presentan tendencias de cambio. Así, la temperatura media anual presenta un incremento de aproximadamente $0,065^{\circ} \mathrm{C} / a n ̃ o$ (Fig. 1A), mientras que la precipitación anual presenta una disminución aproximada de $16 \mathrm{~mm} / \mathrm{año} \mathrm{(Fig.} \mathrm{1B).}$

Figura 1

A) ESTACIÓN INFIERNILLO: TEMPERATURA MEDIA ANUAL ENTRE 1968 Y 2010; B) ESTACIÓN INFIERNILLO: PRECIPITACIÓN ANUAL ENTRE 1968 Y 2010.

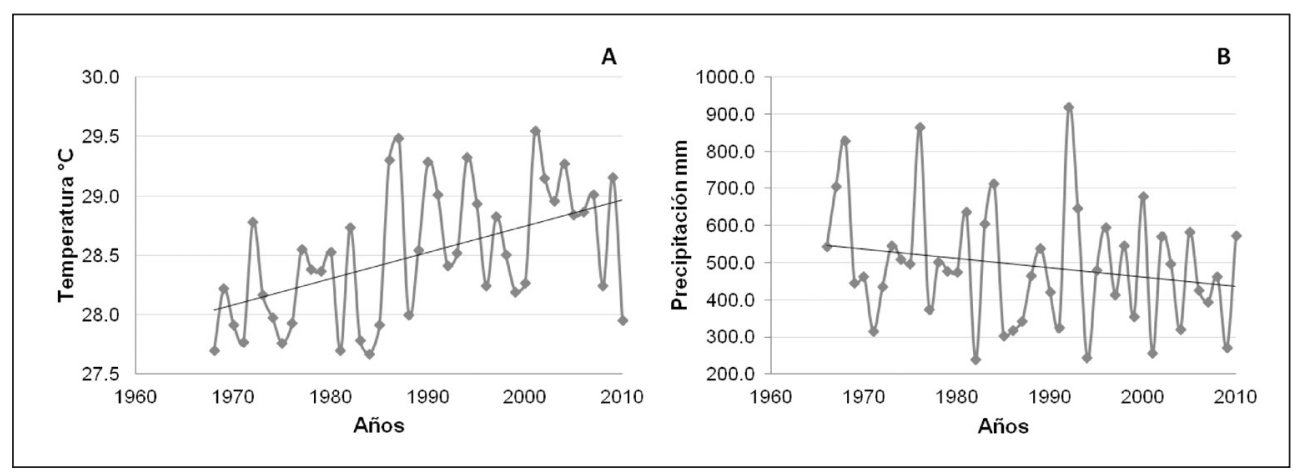

Fuente: Comisión Federal de Electricidad, 2010.

Para el caso de estudio de El Salvador se cuenta con información de temperatura y precipitación de las tres últimas décadas correspondiente a la Estación Cerrón Grande (Departamento de Cabañas), del Sistema Nacional de Estudios Territoriales (SNET). La figura 2A muestra la tendencia de la temperatura, que desde 1974 hasta el año 2010 denota un ligero ascenso, con un promedio aproximado de $0,05^{\circ} \mathrm{C} / \mathrm{año}$. La figura $2 \mathrm{~B}$ muestra la precipitación promedio anual para el mismo período de tiempo, mostrando una tendencia de leve ascenso, relacionada con varios EHE, tales como Fifi (1974), Paul (1982), Adrian y Stan (2005), Ida (2009) y Agata, Alex y Mattew (2010). Además, de acuerdo al SNET, para la última década se ha observado la concentración de mayor cantidad de lluvia en períodos muy cortos de tiempo (MARN, 2011). 
Figura 2

A) ESTACIÓN CERRÓN GRANDE: TEMPERATURA MEDIAANUAL ENTRE 1974 Y 2010; B) ESTACIÓN CERRÓN GRANDE: PRECIPITACIÓN ANUAL ENTRE 1974 Y 2010

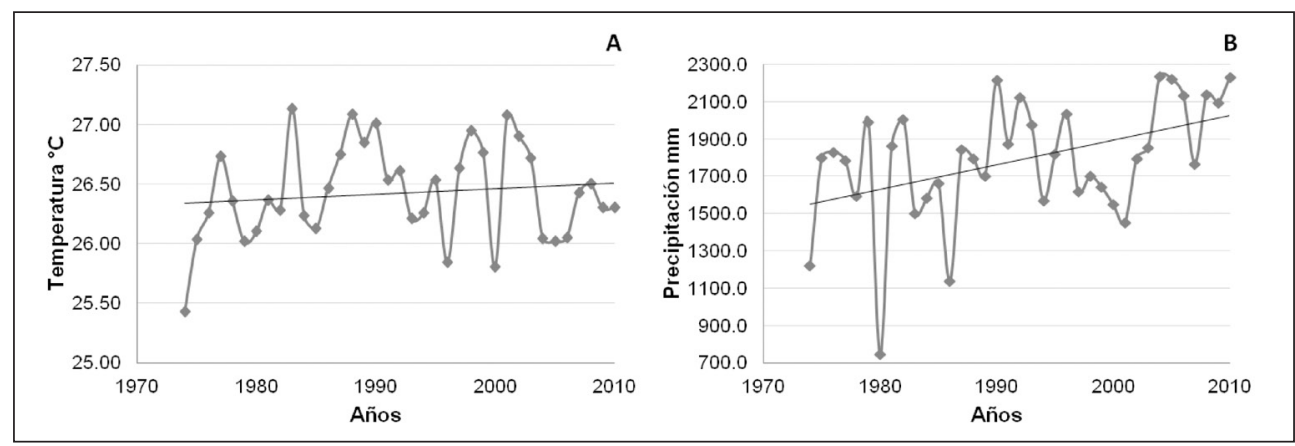

Fuente: SNET 2010

\section{Metodología}

Se utilizó el análisis cualitativo aplicando dos herramientas distintas: grupos focales ${ }^{1}$ y entrevistas semi-estructuradas dirigidas a informantes clave. Esta fase de recopilación de información se desarrolló simultáneamente en los dos ámbitos de estudio entre los meses de enero y julio de 2011.

Los grupos focales se iniciaron explicando a los participantes, de una forma general, los objetivos de la investigación, con el fin de no condicionar sus respuestas. En primer lugar, se expusieron e identificaron los principales problemas relacionados con el cambio climático en cada localidad; una vez identificados los problemas y riesgos se trabajó sobre las acciones y estrategias que la población estaba desarrollando, diferenciando entre aquellas que responden a iniciativas personales, colectivas o canalizadas a través de organizaciones no gubernamentales (ONG's) o instituciones. Posteriormente se abordaron las repercusiones a diferentes escalas de los principales riesgos identificados y el impacto local de los programas y políticas mencionados con anterioridad.

En el caso de México participaron 31 personas ( 25 hombres y 6 mujeres), todos miembros del ejido y propietarios de tierra a excepción de uno, de ellos, que es arrendatario. Por su parte, en El Salvador participaron 42 personas ( 28 hombres y 14 mujeres), residentes en el Área Natural de Cinquera, de los cuales 26 son propietarios de tierra y 16 son arrendatarios.

La información obtenida de los grupos focales fue contrastada y complementada a través de entrevistas semi-estructuradas dirigidas a informantes clave. Para la realización de las entrevistas se formuló un pequeño guión orientativo, que incluía una serie de preguntas abiertas sobre los impactos locales del cambio climático y las estrategias desarrolladas por la pobla-

1 Los grupos focales son una herramienta mediante la cual un grupo de participantes discute sobre un tema específico determinado previamente por los investigadores. La actividad grupal se focaliza en torno a preguntas hechas por los investigadores al grupo. Los investigadores estimulan la participación de todos los miembros del grupo, intercambiando opiniones, anécdotas y puntos de vista. Esta técnica constituye una excelente herramienta para conocer las experiencias, opiniones y preocupaciones de grupos de población sobre un tema determinado (Kitzinger y Barbour, 1999). 
ción en respuesta a los cambios, así como sobre su opinión acerca de los distintos programas y políticas gubernamentales. Con estas entrevistas dirigidas se perseguía recabar reflexiones y opiniones de los entrevistados en relación con las cuestiones recién mencionadas.

\section{Resultados}

\subsection{El caso del Ejido Ichamio, Michoacán (México)}

El estado de Michoacán presenta un alto grado de marginación social y es uno de los estados mexicanos con mayor atraso educativo, ocupando el lugar 30 (de 32) a nivel nacional (CONAPO, 2008). El municipio de La Huacana, donde se localiza el área de estudio, es una zona con alta marginalidad, una fuerte dispersión de la población, un bajo uso productivo del suelo y la ausencia de sistemas de riego. En esta zona el principal riesgo para la actividad productiva son las sequías, ya que se trata de una región en donde las temperaturas son altas (temperatura media anual de aproximadamente $28^{\circ} \mathrm{C}$ ) y hay poca disponibilidad de agua (precipitación promedio anual de 400-500 mm). Al mismo tiempo se trata de una región con

Figura 3

ESTRATEGIAS DE ADAPTACIÓN IDENTIFICADAS POR LOS AGRICULTORES EN MÉXICO. ORIGEN DE LAS INICIATIVAS E INTERRELACIONES EXISTENTES

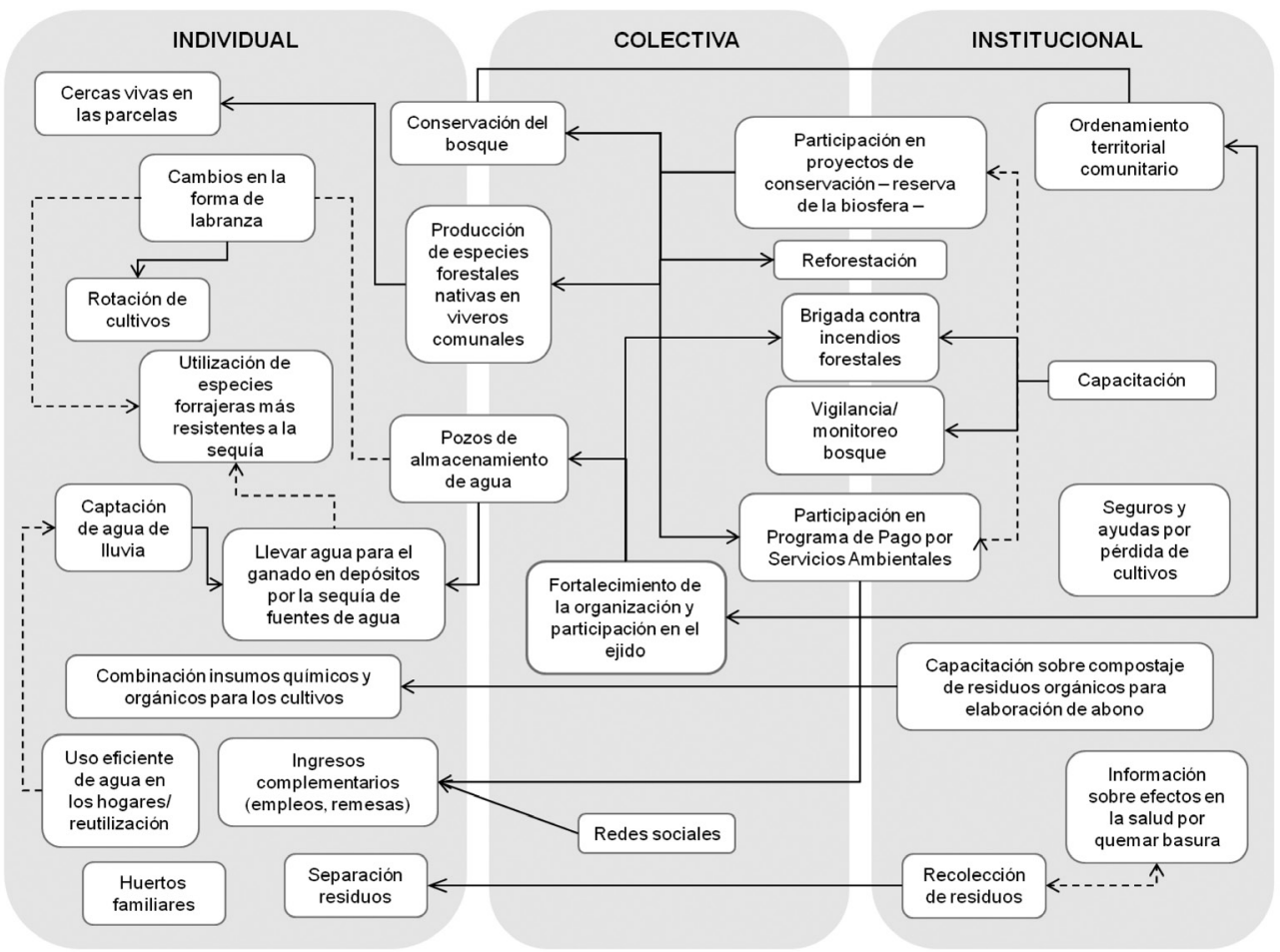

Fuente: Elaboración propia. 
un alto valor ecológico, cuya principal presión es el avance de la ganadería en detrimento del bosque tropical caducifolio (Campos y Boada, 2007).

En el ejido de Ichamio las principales preocupaciones relacionadas con el cambio climático se centran en cuestiones agroambientales, entre las cuales destaca la gestión de los recursos hídricos, la fertilidad y la erosión del suelo y la prevención de la deforestación. En este sentido, durante el taller los participantes, que perciben desde hace años los riesgos de la variabilidad climática, mencionaron una serie de acciones que han estado llevando a cabo, en especial en relación al sector agrícola y forestal. En la figura 3 se presentan las estrategias mencionadas por los participantes en el taller, organizadas en función del origen de las diferentes iniciativas: individuales, colectivas e institucionales. Además, se han establecido las principales relaciones directas e indirectas entre las mismas, permitiendo así explorar la articulación de las respuestas a escala local.

\subsubsection{Estrategias individuales y colectivas}

Entre las estrategias mencionadas sobresalen las que tienen por objeto sortear la incertidumbre de la variabilidad climática y asegurar la producción agrícola y, por ende, la seguridad alimentaria. Debido a los cambios en el inicio de la época de lluvias (mediados de junio), así como en la intensidad y frecuencia de las mismas, el ciclo de cultivo del maíz se ha atrasado o adelantado para ajustar los cultivos a dichos cambios y asegurar así su éxito. Por otro lado, en los últimos años los productores se han preocupado por utilizar menos productos químicos y, en su lugar, estimular la fertilidad del suelo a partir de la rotación de cultivos dentro de las parcelas. También se han introducido pastos forrajeros más resistentes a las sequías para alimentar al ganado. Sobre la producción de ganado, la principal preocupación se refiere a la disponibilidad de agua en las parcelas. Así, el hecho de haberse secado numerosos manantiales en la zona, obliga a los agricultores a transportar agua diariamente en sus vehículos hasta sus parcelas para abastecer al ganado. Por ello, los ejidatarios se han organizado para solicitar una subvención para la construcción de un pozo comunitario que les permitirá canalizar el agua hasta las diferentes parcelas. Los ejidatarios indicaron que habían construido tanques para almacenamiento de agua más grandes en sus hogares, y que además de colectar el agua de lluvia, el agua de uso domestico era re-utilizada en diferentes actividades.

Por otro lado, entre las estrategias mencionadas hay una creciente preocupación por la conservación del bosque. En 2007, la comunidad participó activamente en la definición de la reserva de la biosfera para la conservación del patrimonio natural de la región. Toda vez que una parte importante del ejido forma parte de la misma, se han comprometido a conservar esa parte de su territorio. También se ha creado una brigada contra incendios forestales, de modo que varios miembros de la comunidad realizan recorridos de control y vigilancia por la zona. Dichas acciones, además de haber surgido como medidas de adaptación para hacer frente a los efectos del cambio climático, son también medidas de mitigación a largo plazo, lo cual ha sido señalado también para otros casos en México (Eakin et al., 2006). Esto demuestra claramente las sinergias entre adaptación y mitigación en las acciones desarrolladas a nivel local (Locatelli, 2010; Skutsch y Solís, 2011).

Algunas de estas medidas son, en todo caso, expresión de una preocupación mayor, generalizada, en relación con problemas ambientales no necesariamente circunscritos a los 
efectos negativos del cambio climático, como es el caso de la lucha contra la deforestación y la erosión. Es también importante mencionar que la diversificación de los ingresos de las familias rurales les permite afrontar gastos extraordinarios como los derivados de los impactos del cambio climático; en el caso concreto del municipio de La Huacana, en el que se presenta un índice de intensidad migratoria medio, alrededor de un 10 por ciento de las familias reciben remesas. Durante las entrevistas varias familias confirmaron que recibían ayudas esporádicas de sus familiares desde Estados Unidos de América; sin embargo, debido a la variabilidad entre las cantidades recibidas y la periodicidad de las remesas, sería difícil saber exactamente qué proporción representan sobre el total de los ingresos familiares. Lo que es evidente es que juegan un papel importante en la economía local y contribuyen a relativizar las opciones de adaptación a cambios ambientales.

\subsubsection{Percepción sobre políticas y programas gubernamentales}

Varias de las estrategias identificadas por los agricultores están directamente relacionadas con políticas y programas nacionales de desarrollo agropecuario y forestal que se encuentran en curso, tal y como se detallan en la tabla 2. Actualmente los programas más emblemáticos del ejido son ProÁrbol, diseñado explícitamente para subsidiar a propietarios de áreas forestales en función del número de árboles plantados, y el Pago por Servicios Ambientales (PSA), diseñado para proveer incentivos económicos a los dueños de terrenos forestales para fomentar prácticas de conservación y evitar el cambio de uso del suelo. Los beneficiarios de ambos programas creen que los apoyos obtenidos son claves para complementar sus ingresos, y la percepción general que tienen sobre el programa es positiva, ya que consideran que aun siendo pocos los beneficiarios directos, repercute favorablemente en toda la comunidad. Por el contrario, aquellos que no son beneficiarios ven con recelo los programas y consideran que tendría que establecerse un planteamiento más adecuado que permitiera una participación más amplia, de modo que las ayudas estuvieran mejor distribuidas en el ejido. En este caso, el peligro es que iniciativas como ProÁrbol y PSA puedan ser percibidas desde una perspectiva puramente económica, cuyo único objetivo sea la transferencia de fondos y recursos económicos a la comunidad. Por este motivo, y por la marginación interna hacia los no-propietarios y de ciertos ejidatarios, existe entre los habitantes del ejido una creciente desconfianza y apatía, lo que tiene como consecuencia una débil identificación con estos proyectos. Esto, sin duda, no contribuye a la formación ni al fortalecimiento de capacidades locales para afrontar los efectos adversos del cambio climático. Sin embargo, al margen del programa ProÁrbol, varias personas están realizando acciones de reforestación en sus parcelas agroforestales, utilizando especies locales producidas en sus hogares o en el vivero comunitario. Entre las especies producidas la más importante es Leucaena leucocephala, por tratarse de una planta de crecimiento rápido, habitual en la formación de cercas vivas y en varias zonas de la localidad.

Adicionalmente, a partir de las entrevistas fue posible identificar los principales problemas de origen externo que pueden afectar el desarrollo de capacidades locales para la adaptación al cambio climático. Entre esos problemas destacan los siguientes: i) la deficiente operatividad de algunos programas de gobierno, que se refleja en el escaso personal destinado para ejecutar diferentes programas de desarrollo, lo que se hace más evidente teniendo 
Tabla 2

POLITICAS PÚBLICAS RELEVANTES ORIENTADAS A LAADAPTACIÓN AL CAMBIO CLIMÁTICO

\begin{tabular}{|c|c|}
\hline \multicolumn{2}{|c|}{ POLÍTICAS Y PROGRAMAS } \\
\hline MÉXICO & EL SALVADOR \\
\hline $\begin{array}{l}\text { Programa de Apoyos Directos al Campo (PROCAMPO): Se creó en } \\
1993 \text { como un mecanismo de transferencia de recursos para compensar a } \\
\text { los productores mexicanos por los efectos negativos del Tratado de Libre } \\
\text { Comercio de América del Norte (TLC). Ha presentado problemas de co- } \\
\text { rupción en la implementación, concentrando muchas veces las ayudas } \\
\text { en un número reducido de productores en ciertas regiones. }\end{array}$ & $\begin{array}{l}\text { Plan de Agricultura Familiar y Emprendedurismo rural para la Seguri- } \\
\text { dad Alimentaria (PAF). El PAF incluye tres programas, orientados a dos } \\
\text { tipos de agricultores El primero está dirigido a mejorar la seguridad ali- } \\
\text { mentaria de los productores de subsistencia, provee de asistencia técnica } \\
\text { a los agricultores, además de proporcionar un paquete de insumos para } \\
\text { cada temporada agrícola que incluye semilla certificada de maíz y frijol, } \\
\text { además de fertilizantes }{ }^{1} \text {. }\end{array}$ \\
\hline $\begin{array}{l}\text { Seguros agropecuarios: } \\
\text { - AGROASEMEX: Empresa estatal que ofrece seguros agrícolas con- } \\
\text { vencionales (para agostaderos y para zonas de producción) y seguros } \\
\text { catastróficos para EHE. } \\
\text { - Fondo de Desastres Naturales (FONDEN): provee fondos ante los } \\
\text { daños ocasionados a la población damnificada y a la infraestructura } \\
\text { pública con motivo de desastres naturales imprevisibles. }\end{array}$ & Seguro Agropecuario (aún no implementado). \\
\hline $\begin{array}{l}\text { Proyecto de conservación de la biodiversidad gestionado por comunida- } \\
\text { des e indígenas de los estados de Oaxaca, Michoacán y Guerrero (COIN- } \\
\text { BIO). Esta iniciativa comenzó en } 2003 \text { y desde entonces ha sido un } \\
\text { importante mecanismo de asistencia técnica a las comunidades locales². }\end{array}$ & $\begin{array}{l}\text { Proyecto de Administración y Consolidación de Áreas Protegidas } \\
\text { (PACAP). El PACAP es un proyecto formulado para lograr el fortale- } \\
\text { cimiento del Sistema de Áreas Naturales Protegidas (SANP) mediante } \\
\text { el desarrollo de un programa piloto que consolida dos áreas naturales } \\
\text { protegidas claves del país: el Lago Guija-San Diego-La Barra y la Bahía } \\
\text { de Jiquilisco }{ }^{3} \text {. }\end{array}$ \\
\hline $\begin{array}{l}\text { ProÁrbol: programa federal de apoyo que otorga ayudas a los poseedores } \\
\text { y propietarios de terrenos para realizar acciones encaminadas a proteger, } \\
\text { conservar, restaurar y aprovechar de manera sustentable los recursos } \\
\text { en bosques, selvas y zonas áridas de México. Diseñado explícitamente } \\
\text { para proporcionar subsidios a los propietarios, ofrece ayudas directas de } \\
\text { acuerdo al número de árboles plantados en los terrenos agrícolas. }\end{array}$ & $\begin{array}{l}\text { Bono Forestal. Iniciado en } 2003 \text {, y dirigido a estimular la producción } \\
\text { forestal, subvenciona a los propietarios de más de una hectárea, para el } \\
\text { establecimiento de plantaciones forestales }{ }^{4} \text {. }\end{array}$ \\
\hline \multicolumn{2}{|l|}{$\begin{array}{l}\text { Programa de Desarrollo Forestal Comunitario (PROCYMAFII). Asiste } \\
\text { a ejidos y comunidades forestales en regiones prioritarias, otorgando } \\
\text { apoyos económicos directos a ejidos y comunidades para que realicen } \\
\text { actividades que fortalezcan su planificación y organización social, estu- } \\
\text { dios de asistencia técnica, talleres y cursos de capacitación para mejorar } \\
\text { el aprovechamiento de sus recursos forestales }{ }^{2} \text {. }\end{array}$} \\
\hline $\begin{array}{l}\text { Áreas Naturales Protegidas ANP. } \\
\text { En México, el instrumento de política ambiental con mayor definición } \\
\text { jurídica para la conservación de la biodiversidad son las Áreas Protegi- } \\
\text { das. Actualmente existen } 174 \text { ANP de carácter federal, que representan } \\
\text { algo menos del 13\% de la superficie total del país. }\end{array}$ & $\begin{array}{l}\text { Sistema Nacional de Áreas Naturales Protegidas. } \\
\text { Las áreas naturales protegidas constituyen la figura jurídica de protec- } \\
\text { ción de la biodiversidad. El Sistema está compuesto por } 118 \text { áreas, que } \\
\text { suman un 3,6\% de la superficie del país }{ }^{3} \text {. }\end{array}$ \\
\hline $\begin{array}{l}\text { Pago por Servicios Ambientales (PSA): diseñado para proveer incenti- } \\
\text { vos económicos a los dueños de terrenos forestales (ejidos, comunidades } \\
\text { y pequeños propietarios) para apoyar las prácticas de conservación y evi- } \\
\text { tar el cambio de uso del suelo en los bosques. Existen cuatro modalida- } \\
\text { des de PSA en México: hidrológicos, conservación de la biodiversidad, } \\
\text { captura de carbono y actividades silvoagrícolas). }\end{array}$ & $\begin{array}{l}\text { Pago por servicios ambientales (PSA). Ha sido aplicado en casos piloto } \\
\text { a través de proyectos financiados por la cooperación externa, para in- } \\
\text { centivar la protección de microcuencas mediante la reforestación y la } \\
\text { adopción de prácticas de conservación de suelos }{ }^{5} \text {. }\end{array}$ \\
\hline
\end{tabular}

1/ Ministerio de Agricultura y Ganadería. 2010. Plan de Agricultura Familiar y Emprendedurismo Rural para la Seguridad Alimentaria. Documento del Plan.

2/ Soberanes, M. y M.J. Burgin. 2009. Catastro de políticas y programas de desarrollo rural en México. Working paper. Rimisp -Centro Latinoamericano para el Desarrollo Rural.

3/ Banco Mundial. 2007. Proyecto de Administración y Consolidación de Áreas Protegidas (PACAP). Página web del Banco Mundial para el medio ambiente.

4/ Ministerio de Agricultura y Ganadería. 2003. Informe de Coyuntura. Programa bono forestal de El Salvador.

5/ Pérez, C. 2004. Pago por servicios ambientales a nivel municipal en Honduras, El Salvador y Nicaragua. Informe anual del Programa para la agricultura sostenible en laderas centroamericanas. 
en cuenta la amplia complejidad y dimensión territorial; ii) el planteamiento de proyectos ambientales que no apuntan a las necesidades reales de las comunidades, motivando que algunos de los programas planteados por las instituciones sean percibidos como ajenos a las preocupaciones locales; iii) las restricciones de manejo adaptativo en algunas políticas (por ejemplo PROCAMPO; véase Tabla 2), ya que en ocasiones se da pie a la perversión de solicitar subvenciones para la introducción de ciertos cultivos aún a sabiendas de la limitada posibilidad de éxito de esas plantaciones; iv) la falta de seguimiento o monitoreo que garantice la idoneidad de las prácticas forestales empleadas (por ejemplo plantaciones de ProÁrbol y PSA); v) el solapamiento y la contradicción entre las políticas y los programas existentes; las contradicciones más comunes se producen entre las políticas de conservación y las políticas de desarrollo agropecuario; y vi) la nula o poca continuidad, planificación y coordinación a largo plazo entre alcaldías, gobiernos estatales y federales en el desarrollo de los programas.

En términos generales es posible resaltar que en la región, y específicamente en el ejido, las acciones de adaptación identificadas han surgido como respuestas puntuales a problemas concretos, pero sin formar parte de estrategias más amplias y a largo plazo. Las iniciativas lideradas y dirigidas por los actores locales incluyen una implantación geográfica (local versus regional/suprarregional), una serie de conocimientos locales (empíricos versus técnicocientífico) y son una pieza clave en la gobernanza local (apropiación versus imposición). Por este motivo se evidencia que los programas ambientales procedentes de instancias gubernamentales deberían presentar un mayor nivel de adaptación a las características propias de los ámbitos de intervención.

\section{2. Área Natural de Cinquera (El Salvador)}

El Área Natural de Cinquera (ANC), es una zona montañosa de 5.500 ha, ubicada en un rango de elevación que oscila entre 200 y 754 m, compartida por más de cien propietarios en cinco municipios predominantemente rurales. Posee una topografía fuertemente accidentada, con pendientes que oscilan entre un 5 y un $75 \%$, y una temperatura media anual de $27^{\circ} \mathrm{C}$. El ANC se ubica en una de las zonas con mayor concentración de pobreza; más del $50 \%$ de la población se encuentra en condiciones de pobreza extrema y pobreza relativa (FLACSO, 2005). Se trata de una población vinculada estrechamente a la actividad agropecuaria. Un $75 \%$ del área natural se ubica dentro de los municipios de Cinquera y Tenancingo, cuya población se dedica en su mayoría a la agricultura de subsistencia. Solo el 15\% del bosque es gestionado en forma colectiva, a través de una organización local fuertemente consolidada, la Asociación para la Reconstrucción y Desarrollo Municipal (ARDM), a través de la cual se han logrado acuerdos colectivos para la protección del bosque con buena parte de los propietarios. Sin embargo, uno de los problemas identificados por dicha organización local es la presión por el cambio de uso del suelo, que además de la pérdida de cubierta forestal, provoca la erosión de los suelos a causa de la siembra de cultivos anuales en laderas y sin prácticas de protección.

La ARDM ha orientado su trabajo en la búsqueda de alternativas económicas que permitan la protección y uso sostenible del bosque, así como de actividades complementarias a la agricultura de subsistencia. La población valora el bosque por considerarlo clave para la protección de caudales. 
La zona es abastecida por dos ríos, que disminuyen considerablemente su caudal en la época seca, situación que se ha ido acentuando en los últimos años. La población local asocia claramente el cambio climático a la mayor ocurrencia de EHE; particularmente, se refirieron la tormenta tropical Ida, que en noviembre de 2009 afectó enormemente a la zona. Así mismo, consideran la reducción de caudales durante la época seca y la sequía como otro de los problemas vinculados al cambio climático. A diferencia del caso mexicano, el ANC no está ubicado en las zonas con mayor incidencia de sequías. Sin embargo, durante la época de lluvias se presenta el fenómeno conocido como «canícula», marcado por varios días sin lluvia e incremento de la temperatura; este patrón, que generalmente ocurre durante la segunda quincena de julio y la primera de agosto y con una duración de entre 5 y 10 días, se está viendo acentuado en los últimos años, afectando en consecuencia a la actividad agrícola.

\subsubsection{Estrategias individuales y colectivas}

La figura 4 muestra las estrategias de adaptación identificadas durante los talleres. Se presentan de acuerdo a la iniciativa de su origen (individual, colectiva o institucional), señalando las vinculaciones que se dan entre las estrategias desde los distintos niveles. Una

Figura 4

ESTRATEGIAS DE ADAPTACIÓN IDENTIFICADAS POR LOS AGRICULTORES EN EL SALVADOR. ORIGEN DE LAS INICIATIVAS E INTERRELACIONES EXISTENTES

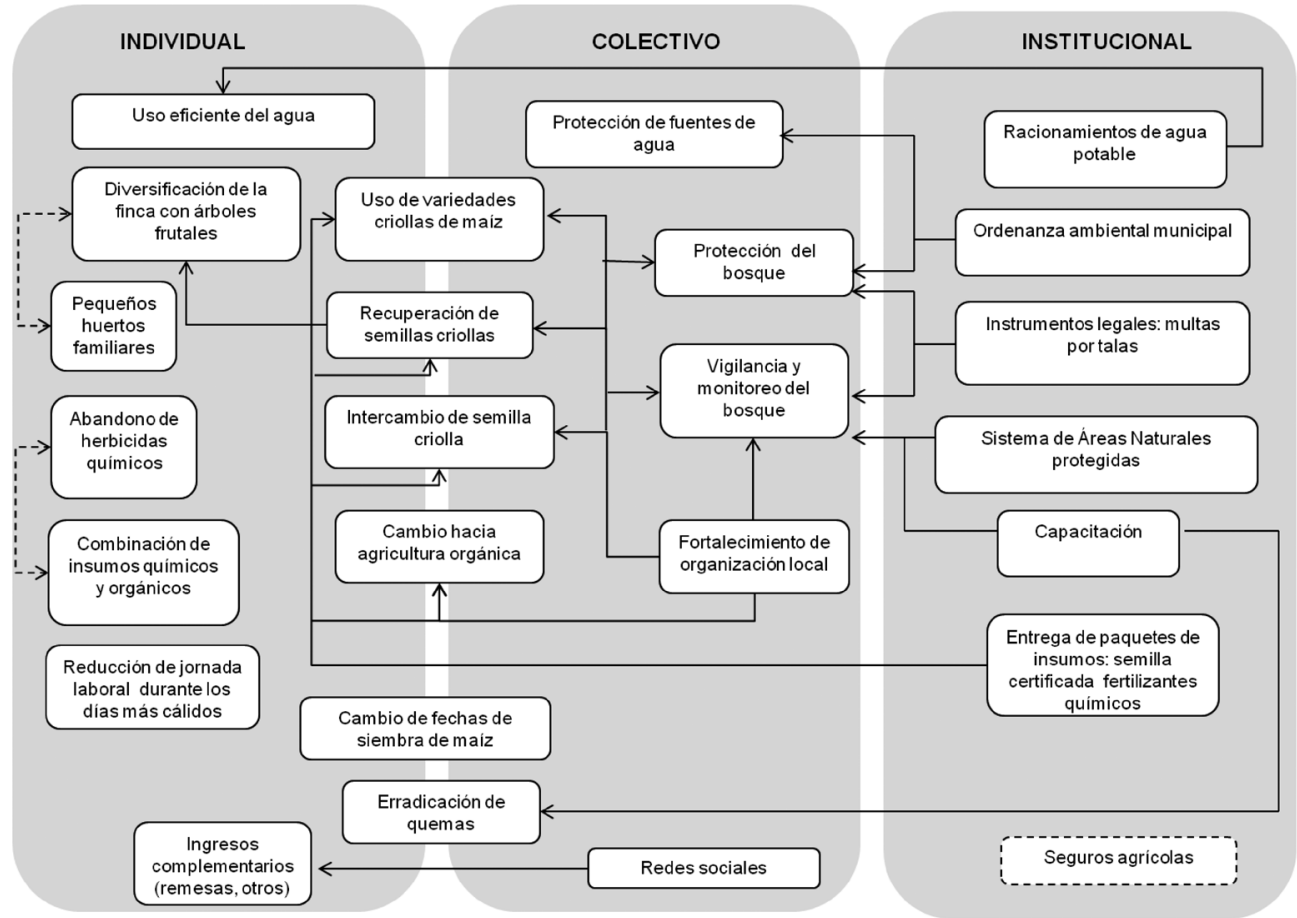

Fuente: Elaboración propia. 
medida tomada en los dos últimos años consiste en retrasar la fecha de siembra de maíz (que tradicionalmente se hace a principios de mayo), esperando el inicio de la temporada de lluvias. Algunos entrevistados mencionaron también una reducción de la jornada de trabajo en los días de más calor. Ciertas unidades productivas agrícolas enfocan sus iniciativas a la diversificación de cultivos asociados al maíz y al frijol. Los asistentes a los talleres mencionaron que es importante incluir en la parcela agrícola, además del maíz, cultivos como hortalizas y, en algunos casos, frutales, que pueden ayudar a hacer frente a eventuales pérdidas por sequías o por fuertes lluvias (ver figura 4).

Se menciona también un cambio de prácticas hacia la agricultura sostenible, a través de la promoción de la agricultura orgánica. Las razones argumentadas por los campesinos para la sustitución de agroquímicos tienen que ver con el reconocimiento expresado acerca del daño que producen los productos químicos sobre la salud y el medio ambiente; pero además, y quizás más importante, la reducción de los costos en la producción derivada de este cambio. Los asistentes mencionaron que en el año 2007 iniciaron un proceso de «volver a la agricultura de nuestros abuelos», refiriéndose a la agricultura con prácticas tradicionales que no incluían el uso de agroquímicos y semillas mejoradas promovidas por la revolución verde. Para los agricultores entrevistados este proceso se desarrolla con la metodología de «campesino a campesino», de tal forma que son ellos mismos los que lo promueven, apoyados desde el inicio por un proyecto de cooperación internacional que fue gestionado por la ARDM. Los entrevistados consideran este proceso muy positivo, si bien su ritmo de implantación está resultando muy lento.

En cuanto a la protección del bosque, las estrategias son colectivas y apoyadas por instrumentos legales a escala local, tal como una ordenanza municipal decretada en 1998, que prohibió las talas y logró erradicar las quemas. También consideran clave la vigilancia realizada por un equipo de cuatro guardabosques contratados por la organización local; se menciona además un reducido apoyo gubernamental a escala nacional.

En el nivel colectivo, queda claro que las estrategias en el ANC apuntan en dos sentidos: uno, el de la protección del bosque; y otro, el de la promoción del cambio hacia la agricultura orgánica, una estrategia con la que los participantes consideran que están contribuyendo a la mitigación de cambio climático. La organización local busca en la agricultura orgánica la disminución de costos y la sostenibilidad de la producción. Vinculada a esta estrategia, está también la de recuperación de semillas criollas. Aunque con estas actividades se pretende avanzar hacia prácticas propias de una agricultura sostenible y de reducción de costes de producción, desde las comunidades locales se valoran también sus efectos, a más largo plazo, en la mitigación del cambio climático, coincidiendo así con lo señalado por algunos autores sobre las ventajas de este tipo de agricultura para la mitigación de gases de efecto invernadero (véase Smith et al., 2008).

De las manifestaciones de los participantes en los talleres se deduce también la importancia que presenta el acceso a ingresos procedentes de actividades no agrícolas, como el turismo rural, el comercio informal o las remesas provenientes de familiares residentes en el extranjero. Estos ingresos posibilitan una menor dependencia respecto de la agricultura y de los efectos de la variabilidad climática en la actividad agrícola. 


\subsubsection{Percepción sobre las políticas y programas gubernamentales}

En los últimos años están surgiendo diferentes políticas y programas que, de forma implícita, tienden a fortalecer la capacidad de adaptación de la población en el sector agrícola, tal como se muestra en la tabla 2. Estas medidas se caracterizan por surgir de forma aislada, con escasa o nula coordinación con otras instituciones, con impactos a corto plazo y, debido a la escala misma a la que van dirigidas (nacional), no toman en cuenta las estrategias que la población local está desarrollando.

Las estrategias de adaptación surgidas desde el ámbito local en el ANC, tanto a escala individual como colectiva, están directamente vinculadas a su problemática agroambiental y socioeconómica, por lo cual es evidente que algunas de estas políticas y programas tienen el potencial de fortalecer las estrategias de adaptación en las parcelas agrícolas, favoreciendo, en consecuencia la capacidad de adaptación de la población. El Programa de Agricultura Familiar (PAF) del Ministerio de Agricultura y Ganadería ha sido el programa más mencionado (ver tabla 2), siendo su objetivo principal contribuir a la seguridad alimentaria a través del fortalecimiento de la agricultura de subsistencia. La estrategia ha consistido en proveer a agricultores de la región de un paquete de insumos al inicio de la temporada agrícola, incluyendo semilla certificada y fertilizantes químicos. Esta estrategia, en el caso de Cinquera, está entorpeciendo las iniciativas locales de promoción de agricultura orgánica y el uso de semillas criollas. La opinión individual de varios agricultores respecto del PAF es positiva, porque les provee de insumos, pero la organización loca considera que la estrategia no está orientada de cara a la sostenibilidad, creando vínculos de dependencia por parte de los agricultores.

En cuanto a las políticas gubernamentales para protección del bosque desde el nivel institucional, tanto nacional como local, tienen un carácter esencialmente punitivo. Si bien se ha hablado de incentivos y programas de pago por servicios ambientales, la realidad es que hasta ahora estos temas no han podido trascender el discurso. Se esperaba que la apuesta del Ministerio del Ambiente y Recursos naturales en El Salvador estuviera orientada a la protección de bosques; sin embargo, la principal acción, aún no implementada, parece estar dirigida a la promoción de prácticas agroforestales, dejando de lado cuestiones relacionadas más directamente con la protección de las áreas de bosque.

En el caso del ANC, la población considera que la cooperación internacional ha tenido mucho impacto en la zona a través de diferentes proyectos. Por ser una de las áreas más afectadas por el anterior conflicto armado, la presencia de la cooperación ha sido significativa; sin embargo, en los últimos años ha disminuido considerablemente. Durante las entrevistas y reuniones de grupos focales la población mencionó el proyecto de agricultura orgánica, antes que las ayudas gubernamentales.

De todo lo anterior se perciben evidentes desfases y descoordinación entre las políticas de las distintas instituciones del sector público. Partiendo de las entrevistas realizadas, se denota que en El Salvador los principales problemas para el desarrollo de una capacidad local para la adaptación al cambio climático son: i) las restricciones normativas y enfoques contradictorios entre las instituciones, priorizando por ejemplo el logro de incrementos inmediatos en la producción agrícola frente a la sostenibilidad de la producción; ii) la nula o escasa continuidad de los programas; iii) el solapamiento entre programas con intervención en una misma 
zona; iv) la ausencia de investigación acerca de las realidades rurales locales; v) la búsqueda de soluciones a corto plazo versus mediano y largo plazo; vi) la total ausencia de incentivos a la conservación dirigidos hacia los agricultores en pequeña escala; vii) la ausencia de objetivos dirigidos al fortalecimiento de la organización local en los programas.

Otra dificultad en la aplicación de estos programas tiene que ver con la presencia de diferentes partidos políticos en los distintos niveles gubernamentales, al igual que la duración de los mandatos de los gobiernos municipales (tres años); se dificulta así la continuidad de las políticas. Además, en El Salvador, después de pasadas dos décadas del conflicto armado, prevalece un clima de polarización partidaria extrema que resulta un factor limitante para el logro de objetivos de desarrollo de las políticas y programas. Así, en el ANC las diferencias partidistas de la población local se matizan con diferencias de enfoques de manejo de los recursos naturales, lo que dificulta el impacto de las iniciativas orientadas a fortalecer la capacidad de adaptación, tanto desde lo local, como por parte de las políticas y programas gubernamentales, así como de proyectos financiados por la cooperación.

\section{DISCUSIÓN: ESTRATEGIAS LOCALES Y POLÍTICAS PÚBLICAS DE DESARROLLO RURAL ¿HACIA UNA MAYOR CAPACIDAD DE ADAPTACIÓN AL CAMBIO CLIMÁTICO?}

El cambio climático tiene una fuerte dimensión institucional (Young, 2002). Esto es evidente, ya que son las instituciones las encargadas de ejecutar las políticas, hacer cumplir las normas y promover las medidas de mitigación y adaptación al cambio climático mediante la aplicación de distintos instrumentos. Algunos autores sugieren que es poco probable que en países en desarrollo las instituciones existentes puedan hacer frente de manera eficiente y equitativa al cambio climático, porque se trata de retos fuera del ámbito de su experiencia (Kane y Yohe, 2000), y porque en general las decisiones se toman a nivel nacional, aunque las consecuencias de esas decisiones son experimentadas a diferentes escalas (nacional, regional y local) (Tompkins y Adger, 2005). Por lo tanto, se sugiere que para la formulación de políticas de adaptación al cambio climático serán necesarias instituciones más flexibles frente a la incertidumbre (Eakin y Luers, 2006), o la creación de otras nuevas destinadas a este fin. Las condiciones económicas constituyen un obstáculo importante en la adaptación (Olsen y Hindi 2002, Hageback et al., 2005), y de forma más amplia, se considera que la adaptación y el desarrollo están claramente vinculados (Narain, 2011).

Es evidente que en los dos casos de estudio aquí analizados se están realizando esfuerzos locales para atenuar los impactos, aunque aún falta mucho por hacer. A pesar de que, aunque no de forma homogénea, en ambos países existen numerosos instrumentos de políticas públicas relacionados con la implementación de decisiones adaptativas al cambio climático, la articulación entre dichos instrumentos es débil o inexistente y por tanto su efectividad y expresión territorial, sobre todo a nivel local, es difusa. Por ello, a pesar de que algunas políticas o programas incluyen entre sus objetivos la adaptación al cambio climático (la mayoría de forma implícita, más que explícita) esto aun no se ve expresado en las localidades rurales en una reducción de su vulnerabilidad. Hasta ahora, parece que las medidas para preparar a la población para enfrentar y adaptarse a las nuevas condiciones han sido insuficientes y débilmente incorporadas en los planes y políticas sectoriales (Manandhar et al., 2011). El reto consiste, por tanto, en la incorporación de dichos ajustes y medidas, en los contextos 
complejos en que se encuentran ambos países, donde concurren importantes cambios políticos, sociales y económicos.

La falta de articulación entre las políticas caracteriza la apuesta al desarrollo rural en México y El Salvador. Así pues, en ambos países se observa, primero, que la aplicación de soluciones se plantea a corto plazo, sin puntos de encuentro para su continuidad en otras de mediano y largo plazo; y, segundo, que se continúan planteando soluciones generales que no responden a las complejas realidades locales. En ambos casos, las políticas de desarrollo agropecuario han apostado por el incremento de la producción y rendimientos a corto plazo frente la disminución de rendimientos a largo plazo, lo cual no garantiza la seguridad alimentaria de las familias rurales. En ambos casos se ha intensificado el uso de agroquímicos y semillas mejoradas que forman parte de los «paquetes agrícolas», que incluyen semillas mejoradas y agroquímicos para su tratamiento, con las que se condicionan las ayudas, provocando, entre otras cosas, la degradación del suelo, y la pérdida de diversidad de variedades de maíces criollos. Adicionalmente se continúa promoviendo el incremento y apertura de nuevas tierras agrícolas en detrimento de bosques y de otras áreas provistas de cubierta vegetal.

Sin duda, alcanzar simultáneamente objetivos tan diversos como crecimiento económico, equidad distributiva, desarrollo social y sostenibilidad ambiental tiene una gran complejidad. Por ello la coordinación entre instituciones es primordial para evitar duplicidad de acciones y dilapidación de los recursos económicos, ya que la adaptación al cambio climático es un problema complejo y multidimensional. La importancia de destacar la necesidad del trabajo intersectorial, multidisciplinario e integral, se debe a que muchas políticas y decisiones que surgen de otros sectores (agua, agricultura, manejo de recursos naturales) pueden influir positiva o negativamente en el fortalecimiento de las capacidades de adaptación. En los casos presentados se considera que las intervenciones políticas no están contribuyendo a fortalecer capacidades locales de adaptación al cambio climático. Esto es crucial, ya que estos programas tienen el potencial de mejorar las habilidades de organización local alrededor de la gestión agroforestal y las acciones de conservación, por lo que su articulación para un uso más eficiente de los recursos económicos y humanos resulta imprescindible. Sin embargo, en los casos de Ichamio y de Cinquera existe un desfase y descoordinación entre políticas que afecta prácticamente a todos los ámbitos en los que tienen lugar las intervenciones públicas. El riesgo consecuente consiste en que estas políticas pierdan credibilidad entre la población, como resultado de las debilidades percibidas en su aplicación.

Pese a existir mecanismos de alerta, de financiación, e incluso de capacitación, parece evidente que existe una fragmentación inter-institucional, pero también poca coordinación y diálogo entre diferentes niveles de gobierno. Esto puede deberse, según Aragón-Durand (2011), a que la mitigación de daños y la adaptación al cambio climático son tratados como temas técnico-ambientales o energéticos, o como tareas de protección civil y de ayuda humanitaria, pero no como parte de la política de desarrollo. Es decir, se hace frente a los costes ocasionados por los daños, pero se invierte y planifica poco en prevenir dichos daños. Esto hace que ante el riesgo de EHE las competencias para desarrollar medidas concretas de mitigación de daños y atender necesidades de adaptación, tengan que ser atendidas por dependencias con diferentes competencias, lo cual provoca duplicidades y descoordinación en la operación de acciones, además de una ejecución deficiente del gasto público destinado a ello. 
En el caso de México las estrategias a nivel colectivo no surgen espontáneamente, sino que funcionan como una interfase de las estrategias que surgen de manera individual o promovidas desde las instituciones como parte de las políticas y programas federales (por ejemplo: PSA), estatales (campañas de salud) o municipales (separación y recogida de residuos). Es destacado el peso que tienen algunas instituciones sobre el territorio, a diferencia de otras menos implantadas, a pesar de estar desarrollando algunos proyectos en la región. En el caso de El Salvador encontramos una situación similar; sin embargo, aquí la cooperación internacional está jugando un importante papel a través de la implementación de proyectos que, sin estar articulados con las políticas y programas gubernamentales, pueden ejercer un impacto mayor a nivel local. Sobre todo debido a la notable ausencia de incentivos dirigidos a los pequeños agricultores en este país. Resulta fundamental mejorar en ambos países los mecanismos para acceder a los beneficios de las políticas y programas, a fin de que resulten más claros (menos burocráticos), y reducir el tiempo de asignación de los recursos, lo cual implicaría una mejora en la gestión de los fondos públicos destinados a disminuir la vulnerabilidad y fortalecer la adaptación. Además, resulta también importante incrementar las inversiones en políticas de prevención, ya que resulta más costoso atender a la población afectada por los desastres que invertir en políticas para reducir la vulnerabilidad y mitigar los efectos del cambio climático (Kelly y Adger, 2000).

Por otro lado, deben mencionarse iniciativas recientes de mitigación del cambio climático, como son los mecanismos de Reducción de Emisiones por Deforestación y Degradación (REDD+). Los mecanismos de REDD+ se visualizan como una oportunidad de financiación directa para las zonas forestales en los países en desarrollo y, por tanto, han despertado mucho interés a nivel mundial. De acuerdo a Corbera et al. (2011), es probable que la estrategia REDD+ en México se estructure en torno al Programa de Pago por Servicios Ambientales (PSA), y en ese sentido merece la pena recordar que, hasta ahora, la evidencia sobre los efectos de PSA sobre la reducción de la deforestación están lejos de ser concluyentes (Muñoz-Piña et al., 2008). En el caso de El Salvador, caracterizado por elevadas tasas de deforestación (FAO, 2009), el Ministerio del Medio Ambiente y Recursos Naturales (MARN) está diseñando el Plan de Restauración de Ecosistemas y Paisajes Rurales, orientado al estímulo del sector agroforestal en zonas vulnerables del país. En ambos contextos, los instrumentos REDD+ pueden financiar actividades en el sector forestal y agroforestal, y cumplir, además de su objetivo global inicial de mitigación del cambio climático, con el objetivo también esencial, aunque a escala local o nacional, de la adaptación.

Como se muestra en ambos casos de estudio, al igual que en otras investigaciones (Eakin y Wehbe, 2009; Mertz et al., 2009), es evidente que desde el nivel local se están desarrollando estrategias importantes de adaptación al cambio climático que surgen como respuestas inmediatas a cambios e impactos relacionados con la variabilidad climática. Debido a que la adaptación es un asunto principalmente local, su eficacia depende del entramado de instituciones y políticas a través de las cuales se estructuren las respuestas a largo plazo (Agrawal, 2008). Así, las políticas que, por ejemplo, faciliten los procesos de diversificación económica sin menoscabar la viabilidad de la producción agrícola no sólo ayudarán a las familias a hacer frente a las incertidumbres ambientales, sino también a darles la seguridad económica necesaria (Eakin, 2005). 


\section{CONCLUSIONES}

En este artículo se ha reflexionado sobre experiencias locales de adaptación al cambio climático en dos comunidades rurales de México y El Salvador. Además, se ha discutido en torno a la articulación entre estas respuestas y estrategias locales de adaptación y las políticas públicas orientadas a la promoción del desarrollo rural y a fortalecer las capacidades locales para la adaptación. A partir de lo anterior, podemos concluir que, a pesar de las diferencias entre ambos países, lo cual podría requerir un análisis más profundo para cada caso, a través de este artículo hemos logrado identificar y explorar: i) diversas iniciativas locales de adaptación que se comparten en diferentes contextos geográficos, que tendrían que fortalecerse; ii) debilidades en la instrumentación y operatividad de planes/programas y políticas a escala local; iii) el potencial de ciertos planteamientos y políticas que, sin embargo, tienen una expresión territorial difusa; y iv) la urgencia de mejorar la velocidad de respuesta ante los riesgos y retos climáticos, ya que en general existe un desfase entre las necesidades locales y las respuestas institucionales.

Es importante resaltar que algunas de las medidas acometidas, tanto a instancias de la población local como de instituciones gubernamentales, se presentan vinculadas al cambio climático aunque su intención primera y directa se relacione con problemas de conservación del bosque o de lucha contra la erosión, o con la preocupación por la incorporación de agroquímicos en la actividad productiva. Se trata, pues, de medidas que conectan con problemas ambientales que trascienden la lucha contra el cambio climático, aunque, como se señaló previamente, algunos autores resaltan el efecto indirecto de mitigación del cambio climático en la agricultura orgánica, por ejemplo. En todo caso, la propia percepción de esas actuaciones es sintomática de la asunción del cambio climático como problema ambiental por parte de las comunidades locales.

Hemos enfocado el análisis en la adaptación de forma separada de la mitigación. Sin embargo consideramos que ambos temas son complementarios en respuesta al cambio climático. Algunas estrategias de adaptación pueden tener impacto en la mitigación de cambio climático y viceversa (Smit et al., 2000); en este artículo hemos enfatizado la adaptación, ya que dadas las condiciones de vulnerabilidad de las zonas rurales de estos países resulta prioritario y urgente. Además, consideramos que el interés desde distintos sectores para mejorar la capacidad de adaptación al cambio climático abre nuevos e importantes espacios para la articulación y complementación de iniciativas orientadas al desarrollo rural.

\section{AGRADECIMIENTOS}

Los autores agradecen a las organizaciones locales, tanto del ejido de Ichamio y del Área Natural de Cinquera, por el apoyo y la colaboración brindada durante la fase de campo de este estudio. Queremos agradecer a Alejandro Velázquez y Martí Boada por sus valiosos comentarios sobre una versión preliminar de este trabajo. Parte del proyecto fue financiado con el apoyo de DEGAPA-IN 307710. Los primeros autores han sido apoyados respectivamente por una beca postdoctoral de la Universidad Nacional Autónoma de México (UNAM) y una beca postdoctoral del Consejo Social de la Universidad Carlos III de Madrid. 


\section{REFERENCIAS}

ADGER, W. N. (2000): «Social and ecological resilience: are they related?» Progress in Human Geography, n 24, 347-364.

ADGER, W. N., ARNELL, N. W. y TOMPKINS, E. L. (2005). «Successful adaptation to climate across scales». Global Environmental Change $\mathrm{n}^{\circ}$ 15, 75-76.

AGUILAR, E., PETERSON, T. C., RAMÍREZ-OBANDO, P., FRUTOS, R., RETANA, J. A., SOLERA, M., SOLEY, J., GONZÁLEZ-GARCÍA, I., ARAUJO, R. M., ROSASANTOS, A. VALLE, V. E., BRUNET, M., AGUILAR, L., ÁLVAREZ, L., BAUTISTA, M., CASTAÑÓN, C., HERRERA, L., RUANO, E., SINAY, J. J., SÁNCHEZ, E., HERNÁNDEZ-OVIEDO, G. I., OBED, F., SALGADO, J. E., VÁZQUEZ, J. L., BACA, M., GUTIÉRREZ, M., CENTELLA, C., ESPINOSA, J., MARTÍNEZ, D., OLMEDO, B., OJEDA, C. E., NÚÑEZ, R., HAYLOCK, M., BENAVIDES, H. y MAYORGA, R. (2005): «Changes in precipitation and temperature extremes in Central America and northern South America, 1961-2003». Journal Of Geophysical Research , nº 110, D23107.

AGRAWAL, A. (2008): The Role of Local Institutions in Adaptation to Climate Change. Social Dimensions of Climate Change Workshop, World Bank; Washington, DC.

ARAGÓN DURAND, F. (2011): «Adaptación al cambio climático y gestión del riesgo de desastres en México: obstáculos y posibilidades de articulación». Cambio climático, amenazas naturales y salud en México. El Colegio de México, Centro de Estudios Demográficos, Urbanos y Ambientales, Centro de Estudios Económicos. p. 131-158.

BERKES, F. y JOLLY, D. (2001): «Adapting to climate change: social-ecological resilience in a Canadian western Arctic community». Conservation Ecology n ${ }^{\circ}$ 5(2), 18. en: http:// www.consecol.org/vol5/iss2/art18.

BRYAN, E., DERESSA, T. T., GBETIBOUO, G. A. y RINGLER, C. (2009): «Adaptation to climate change in Ethiopia and South Africa: options and constraints». Environmental Science and Policy $\mathrm{n}^{\circ}$ 12, 413-426.

CAMPOS, M. y BOADA, M. (2007): «Aplicación de la metodología de Eurosite para la planificación de la gestión de espacios naturales a un caso mexicano». Boletín de la Asociación de Geógrafos Españoles, $\mathrm{n}^{\circ}$ 45, 233-248.

CANNON, T. (1994): Vulnerability Analysis and the Explanation of Natural Disasters. In Varley, A. (Ed.), Disasters Development and Environment, John Wiley, Chichester, pp. 13-30.

CONAPO, (2008): Principales Indicadores Sociodemográficos del estado de Michoacán con base en las elaboraciones del Consejo Nacional de Población, Morelia, Michoacán, México.

CONDE ÁlVAREZ, C., y SALDAÑA-ZORRILLA, S. O. (2007): «Cambio climático en América Latina y el Caribe: Impactos, vulnerabilidad y adaptación». Revista Ambiente y Desarrollo $\mathrm{n}^{\mathrm{o}} 23,23-30$.

CORBERA, E., ESTRADA, M., MAY, P., NAVARRO, G. y PACHECO, P. (2011). «Rights to Land, Forests and Carbon in REDD+: Insights from Mexico, Brazil and Costa Rica». Forests $\mathrm{n}^{\circ} 2,301-342$.

EAKIN, H. (2005). «Institutional Change, Climate Risk, and Rural Vulnerability: Cases from Central Mexico». World Development, nº 33, 1923-1938. 
EAKIN H. y WEHBE M. (2009): «Linking local vulnerability to system sustainability: Two cases from Latin America». Climatic Change, $\mathrm{n}^{\circ}$ 93, 355-377.

EAKIN, H. y LUERS, A. L. (2006). «Assessing the vulnerability of social-environmental systems». Annual Review of Environmental Resources, $\mathrm{n}^{\circ}$ 31, 365-94.

EAKIN H., WEBHE, M., ÁVILA, C., SÁNCHEZ-TORRES, G. y BOJÓRQUEZ-TAPIA, L. A. (2006): A comparison of the social vulnerability of grain peasants in Mexico and Argentina. AIACC Working Paper No. 29. Washington, D. C.

ERIKSEN, S. y SELBOE, E. (2012): «The social organisation of adaptation to climate variability and global change: the case of a mountain farming community in Norway». Applied Geography, no $33,159-167$.

FAO (2009): Situación de los bosques del mundo. 151p.

FLACSO (2005): Mapa de pobreza de El Salvador. Programa El Salvador.

FISCHER. G., SHAH, M., TUBIELLO, F. N. y VAN VELHUIZEN H. S. (2005): «Socioeconomic and climate change impacts on agriculture: an integrated assessment 1990-2080». Philosophical Transactions of the Royal Society B, $\mathrm{n}^{\circ}$ 360, 2067-2083.

HAGEBACK, J., SUNDBERG, J., OSTWALD, M., CHEN, D., YUN, X. y KNUTSSON, P. (2005): «Climate variability and land-us change in Danagou watershed, China-exemples of small-scalle farmers' adaptation». Climatic Change, $\mathrm{n}^{\circ} 72,189-212$.

IPCC. (2007): Fourth Assessment Report. Intergovernmental Panel on Climate Change Secretariat. Geneva, Switzerland.

KANE, S. e YOHE, G. (2000): «Societal adaptation to climate variability and change: an introduction». Climatic Change $\mathrm{n}^{\circ} 45,1-4$.

KELLY, P. M. y ADGER, W. N. (2000): «Theory and practice in assessing vulnerability to climate change and facilitating adaptation». Climatic Change, ${ }^{\circ}$ 47, 325-352.

KITZINGER, J. y BARBOUR, R. (1999): «Introduction: the challenge and promise of focus groups». In Barbour, R. and Kitzinger, J. Eds. (1999): Developing focus group resarch. Politics, theory and practice. SAGE publicaciones. $225 \mathrm{p}$.

LANDA, R., MAGAÑA, V. y NERI, C. (2008): Agua y Clima: elementos para la adaptación al cambio climático. SEMARNAT-CCA-UNAM.

LOCATELLI, B. (2010): Local, Global: Integrating Mitigation and Adaptation. Perspective Forests/Climate Change. $\mathrm{n}^{\mathrm{o}} 3$.

MARN (Ministerio del Medio Ambiente y Recursos Naturales). (2011): Evaluación de daños y pérdidas en El Salvador ocasionados por la Depresión Tropical 12E. Informe preliminar. El Salvador.

MERTZ, O., MBOW, C., REENBERG, A. y DIOUF, A. (2009): «Farmers' Perceptions of Climate Change and Agricultural Adaptation Strategies in Rural Sahel». Environmental Management, $\mathrm{n}^{\circ}$ 43, 804-816.

MORTON, J. F. (2007): «The impact of climate change on smallholder and subsistence agriculture». Proceedings of the National Academy of Sciences of the United Estates, $\mathrm{n}^{\circ} 104$, 19680-19685.

MUÑOZ-PIÑA C., GUEVARA, A., TORRES, J. M. y BRAÑA, J. (2008): «Paying for hydrological services of Mexico“s forests: Analysis, negotiations and results». Ecological Economics, $n^{\circ} 65,725-736$. 
NARAIN, U., MARGULIS, S. y ESSAM, T. (2011): «Estimating costs of adaptation to climate change». Climate Policy, $\mathrm{n}^{\circ} 11,1001-1019$.

O'BRIEN, K. y LEICHENKO, R. (2000): «Double exposure: assessing the impacts of climate change within the context of economic globalization». Global Environmental Change, $\mathrm{n}^{\mathrm{o}} 10,221-232$.

OLSEN, J. e HINDI, M. (2002): «Consequences of climate change for European agricultural productivity, land use and policy». European Journal of Agronomy, n 16, 239-262.

SAMANIEGO, J. (2009): Cambio climático y desarrollo en América Latina y el Caribe: una reseña. Documento de proyecto. Comisión Económica para América Latina y el Caribe (CEPAL), Naciones Unidas. Santiago de Chile.

SKUTSCH, M. y SOLIS, S. (2011): How much carbon does community forestry save? In Skutsch, M. (Ed.) Community forest monitoring for the carbon market: Oportunities under REDD. Earthscan, London, UK.

SMIT, B., BURTON, I., KLEIN, R. Y WANDEL, J. (2000): An anatomy of adaptation to climate change and variability». Climatic Change, $\mathrm{n}^{\circ}$ 45, 223-251.

SMITH, P., MARTINO, D., ZUCONG, C., GWARY, D., JANZEN, H., KUMAR, P., McCARL, B. OGLE, S., O’MARA, F., RICE, C., SHOLES, B., SIROTENKO, O., HOWDEN, M., McALLISTER, T., PAN, G., ROMANENKOV, V., SCHNEIDER, U., SIRINTORNTHEP, T., WATTENBACH, M. y SMITH, J. (2008): «Greenhouse gas mitigation in agriculture». Philosophical transactions of The Royal Society B, $\mathrm{n}^{\circ} 363,789$ 813.

TOMPKINS, E. L., y ADGER, W.N. (2005): «Defining response capacity to enhance climate change policy». Environmental Science \& Policy, ${ }^{\circ}$ 8, 562-571.

TUBIELLO, F. N. y ROSENZWEIG, C. (2008): «Developing climate change impact metrics for agriculture». Integrated Assessment Journal, $\mathrm{n}^{\circ}$ 8, 165-184.

WALKER, B., CARPENTER, S.R., ERIES, J., ABEL, N., CUMMING, G.S., JANSSEN, M., LEBEL, L., NORBERG, J., PETERSON, G.D. y PRITCHARD, R. (2002): «Resilience management in social-ecological systems: a working hypothesis for a participatory approach». Conservation Ecology, n ${ }^{\circ} 6$ en: http://www.ecologyandsociety.org/vol6/iss 1/ art14/print.pdf.

YOUNG, O, R. (2002): The Institutional Dimensions of Environmental Change: Fit, Interplay, and Scale (Global Environmental Accord: Strategies for Sustainability and Institutional Innovation). The MIT press. Cambridge, Massachusetts. 
